\title{
Neoadjuvant Chemotherapy during Pregnancy in Cervical Cancer Patient Followed by Simultaneous Cesarean Section and Radical Hysterectomy: A Case Report
}

\author{
Olga P Matylevich, MD*
}

Department of Gynecologic Oncology, NN Alexandrov National Cancer Centre of Belarus, Belarus

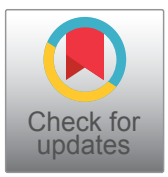

*Corresponding author: Olga P Matylevich, MD, Department of Gynecologic Oncology, NN Alexandrov National Cancer Centre, a/g Lesnoy-2, Minsk, 223040, Belarus, Tel: +375-17-389-9783; +375-29-6119-752

\begin{abstract}
Current recommendations for pregnant women with cervical cancer depend on several factors, such as the stage of the disease, the gestational age at the time of diagnosis, and the patient's desire to maintain the pregnancy. This report describes the case of a 24 weeks pregnant woman with stage IB2 cervical cancer who was successfully treated with three cycles of platinum-based neoadjuvant chemotherapy followed by a simultaneous Caesarean section and radical hysterectomy. Her newborn daughter was completely healthy at birth. As far as we know, this was the first case of cervical cancer during pregnancy to be treated with this approach in Belarus. In the absence of clear guidelines, these patients should be treated after a decision by a multidisciplinary cancer committee, including a gynecologist-oncologist, obstetrician, pathologist and neonatologist, to determine the best treatment option.
\end{abstract}

\section{Keywords}

Cervical cancer, Pregnancy, Neoadjuvant chemotherapy, Simultaneous caesarean section, Radical hysterectomy

\section{Introduction}

Due to the current trend of having children at an older age, the number of pregnancies complicated by cervical cancer is increasing [1]. Radical hysterectomy with pelvic lymph node dissection is currently the standard of care for cervical cancer patients with International Federation of Gynecology and Obstetrics (FIGO) 2018 IA2-IB2 Stages [2,3]. In pregnant women this treatment is possible planning in cases of pregnancy no preserving management. But analysis of the prognosis of cer- vical cancer during pregnancy shows the absence of a negative impact of pregnancy on the patient's outcome [4-6]. Therefore, management with continued pregnancy should be considered initially. According to the recommendations of the International Network on Cancer, Infertility and Pregnancy (INCIP) after the $22^{\text {nd }}$ week of gestation, delayed treatment after delivery with regular follow-up could be initiated. Alternatively, Neoadjuvant Chemotherapy (NACT) could be used to give time to achieve fetal viability by preventing disease progression $[5,7]$. However, data on pharmacokinetics of chemotherapy during pregnancy are scarce and current evidence is based on relatively small case series $[5,8]$. The purpose of this report was to evaluate the outcomes of neoadjuvant chemotherapy during pregnancy followed by Cesarean section and radical hysterectomy in a woman with cervical cancer who had her first pregnancy and had a strong interest in keeping it.

\section{Case Presentation}

A 31-year-old nulliparous woman was referred to the NN Alexandrov National Cancer Centre at 24 weeks of pregnancy with unremarkable medical, surgical, or family history. During prenatal care at the time of 22 weeks' gestation she had presented with abnormal vaginal bleeding. Since the patient was pregnant for the first time, she refused to terminate the pregnancy.

\section{Workup}

Patient underwent extensive workup including gy-

Citation: Matylevich OP (2021) Neoadjuvant Chemotherapy during Pregnancy in Cervical Cancer Patient Followed by Simultaneous Cesarean Section and Radical Hysterectomy: A Case Report. Obstet Gynecol Cases Rev 8:203. doi.org/10.23937/2377-9004/1410203

Accepted: June 18, 2021: Published: June 20, 2021

Copyright: (C) 2021 Matylevich OP. This is an open-access article distributed under the terms of the Creative Commons Attribution License, which permits unrestricted use, distribution, and reproduction in any medium, provided the original author and source are credited. 

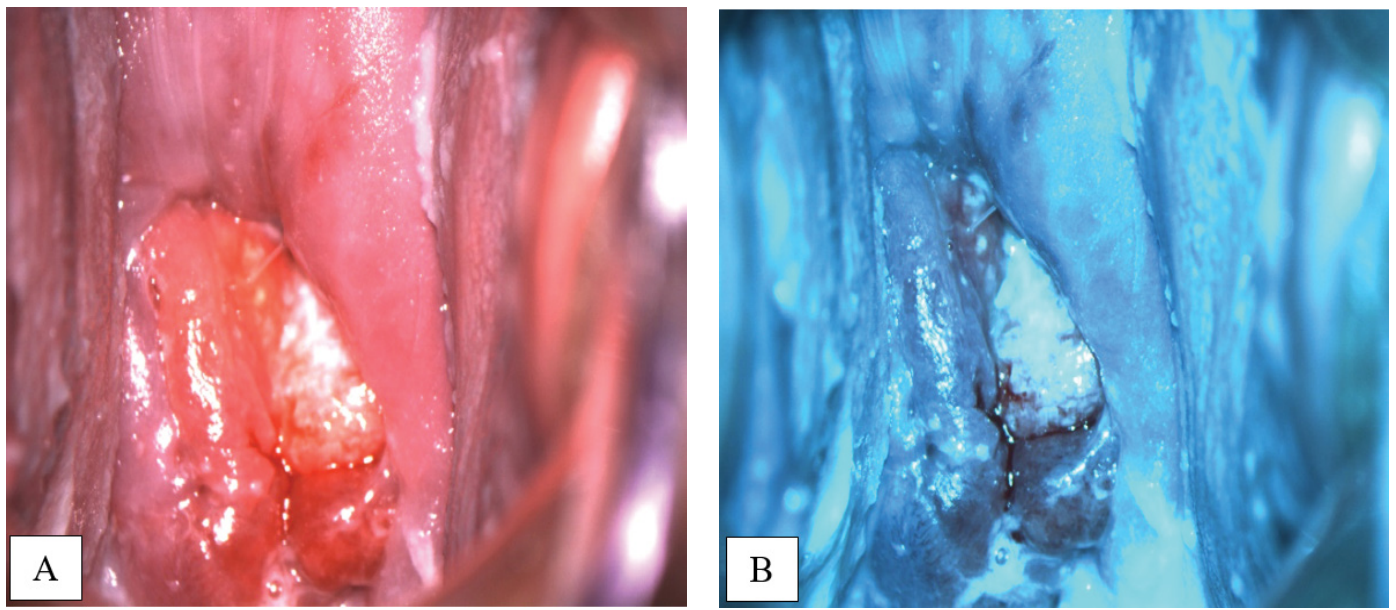

Figure 1: Colposcopy images in white $(A)$ and green $(B)$ filters show tumor on the posterior lip of the cervix.
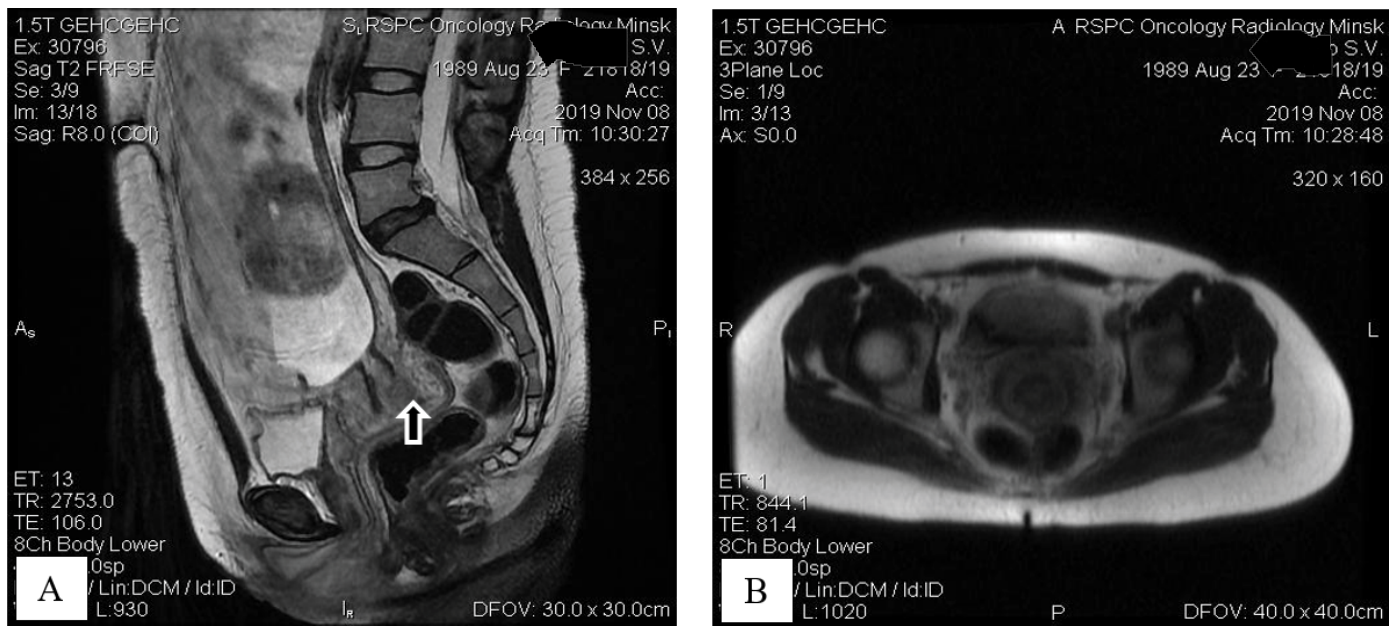

Figure 2: Tumor visualization with sagittal T2-weighted MR image demonstrates intrauterine gestation and cervical mass on the back lip of the cervix with preserved cervical ring (A, arrow); coronal T2W MR view shows no pelvic lymphadenopathy (B).

necologic exam, colposcopy evaluation, pelvic Magnetic Resonance Imaging (MRI) and biopsy of the cervix.

On pelvic examination an exophytic cervical tumor measuring $3 \mathrm{~cm}$ in diameter on the posterior lip of the cervix was found; there were no evidence of parametrial or vaginal involvement. Colposcopy examination was performed, which confirmed the tumor on the posterior lip of the cervix (Figure 1).

Staging studies with pelvic MRI allowed to visualize an intrauterine pregnancy and cervical mass measuring 1.7 by 2.5 by $3.0 \mathrm{~cm}$, mainly on the back lip of the cervix; cervical ring was preserved and there were no suspicious pelvic or inguinal lymphadenopathy (Figure 2).

Histological examination of biopsy of the cervix revealed invasive squamous cell carcinoma, G2, without convincing evidence of lymphvascular space involvement (Figure 3).

Based on these results and according to the 2018 FIGO classification, the patient was diagnosed with stage IB2 invasive squamous cell carcinoma of the cervix in combination with a 24-week pregnancy.

\section{Treatment and follow-up}

The patient's treatment plan was discussed during Multidisciplinary Conference, including a gynecologic oncologist, obstetrician, neonatologist, pathologist, and medical oncologist. Given the patient's strong desire to preserve the pregnancy, to help reach fetal maturity, which is defined as a delivery after 37 weeks' gestational age, platinum-based neoadjuvant chemotherapy was admitted.

Three courses of paclitaxel and carboplatin scheme were performed with 3-weekly interval. Patient tolerated chemotherapy well without any signs of toxicity. The pregnancy continued with no complications too. The efficacy of neoadjuvant chemotherapy was confirmed by colposcopy examination (Figure 4) and pelvic MRI 3 weeks after the completion of the third course of NACT.

At 37 weeks' gestationa Cesarean section was performed using the lower midline skin incision. A female child was delivered. Birth weight of infant was 3380 grams, height - $52 \mathrm{~cm}$; the Apgar score was 8.8 points. After delivery, a radical abdominal hysterectomy (type III, C) [9] was performed with pelvic lymph node dissec- 

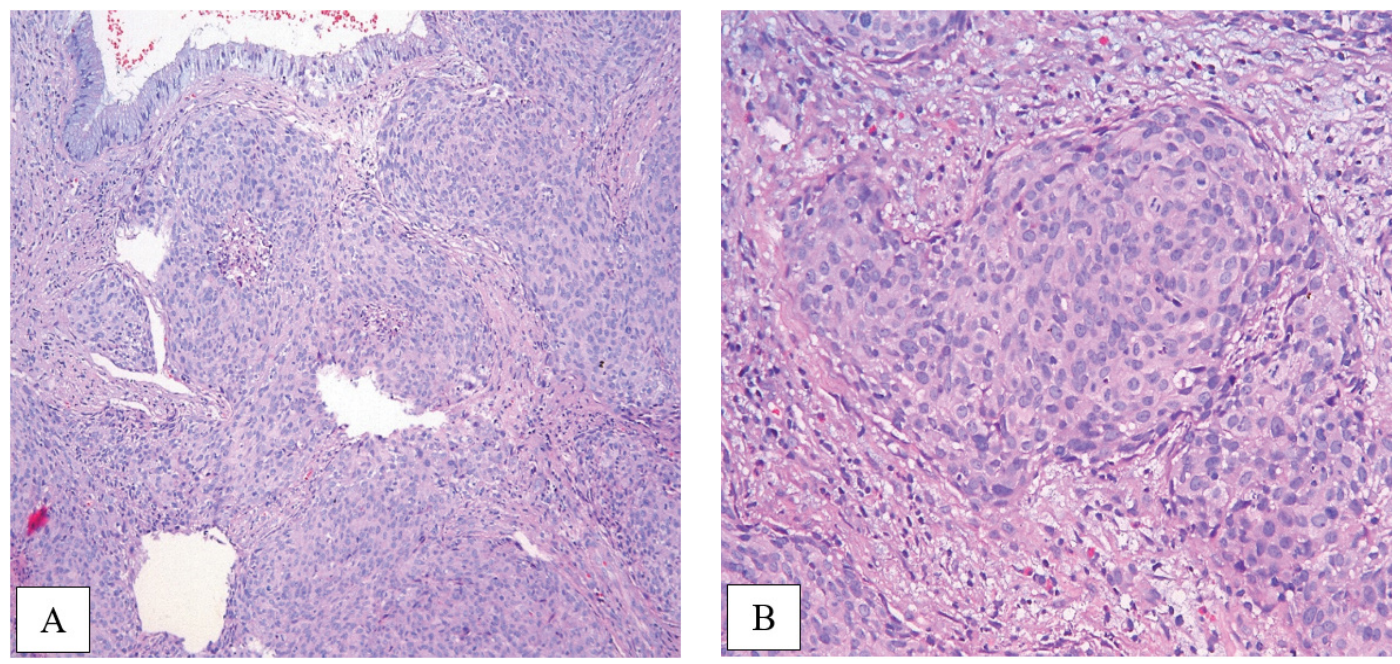

Figure 3: Morphology slides demonstrate invasive squamous cell carcinoma, grade $2(A, \times 10),(B, \times 20)$.
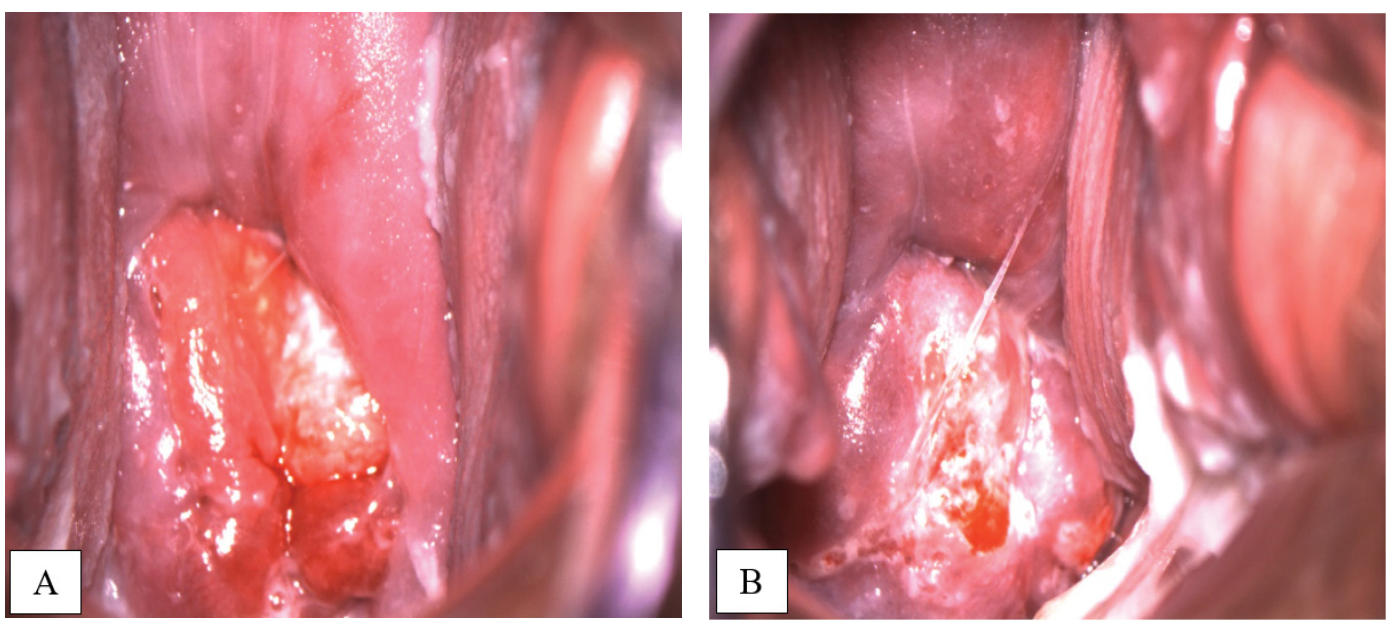

Figure 4: A) Colposcopy examination before NACT in 24 weeks gestation; B) After 3 courses of NACT in 37 weeks gestation.
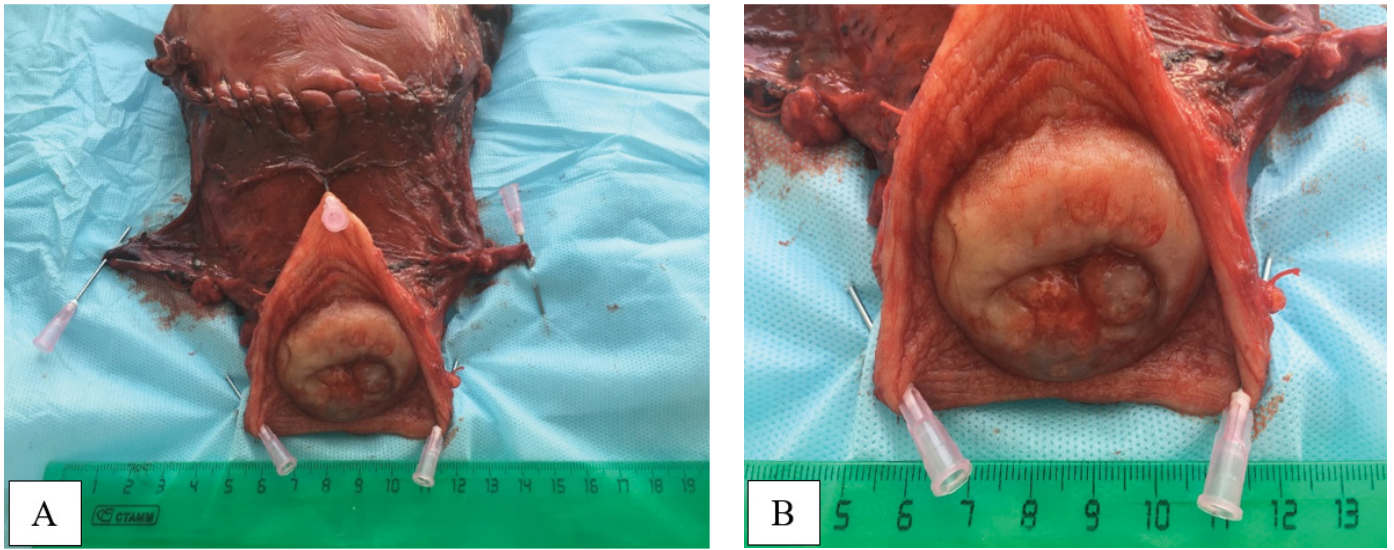

Figure 5: A macroscopic view of hysterectomy specimen with Cesarian scar, parametrial tissues and cervix with vaginal flap $(A, B)$.

tion, bilateral tubectomy, and ovarian transposition. Macroscopically, there are no signs of a tumor of the cervix (Figure 5).

Final pathology report of hysterectomy specimen showed small evidence of invasive squamous cell carcinoma, grade 2, with depth of invasion $3.5 \mathrm{~mm}$ and horizontal spread $3.5 \mathrm{~mm}$. But multifocal invasion of lymphatic vessels was determined. Endometrium with decidual transformation, residual chorionic villi in the area of the placental site were found. The underlying myometrium was with interstitial edema. The both fallopian tubes were of normal structure. Cutting edge of 

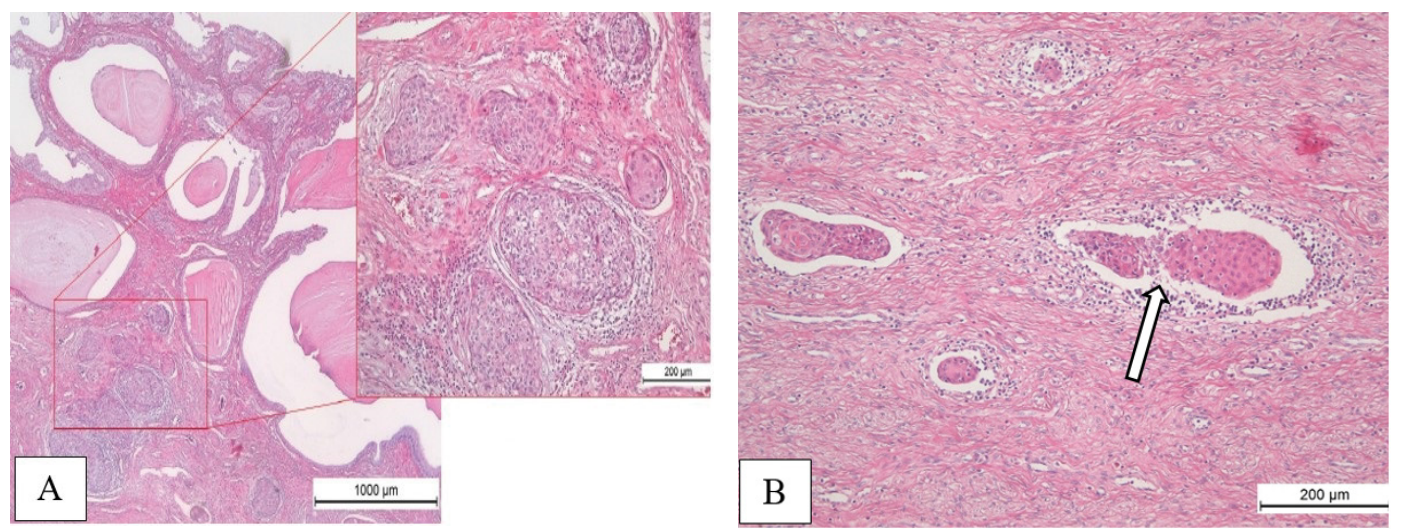

Figure 6: Pathology of hysterectomy specimen. Small focuses of invasive squamous cell carcinoma, depth of invasion 3.5 $\mathrm{mm}(\mathrm{A})$; Tumor emboli in lymphatic vessels ( $B$, arrow).

the vagina had no tumor growth. Pelvic lymph nodes (23) were negative, with foci of endosalpingiosis and subcortical islets of decidual tissue in some of them (Figure 6).

Based on these data and after Multidisciplinary Conference, postoperative course of radiotherapy was admitted. External beam pelvic radiotherapy at a total dose of $44 \mathrm{~Gy}$ fractionated over a period of 31 days was given. She tolerated radiation therapy well and is currently without symptoms of recurrence of her cancer, and radiation induced changes. She is free of the disease at 12 months of follow-up. Her baby is healthy too without any abnormalities.

\section{Discussion}

Cervical cancer is the most common malignant neoplasm diagnosed during pregnancy and, fortunately, most patients are diagnosed having early stages of the disease [5,10-12]. Treatment for cervical cancer during pregnancy depends on a number of factors, such as the stage of the disease (tumor size), lymph node involvement, gestational age, histological subtype, the patient's desire to continue the pregnancy and the desire for the future fertility spearing [11]. It is important to emphasize that during pregnancy the oncological outcome in patients with cervical cancer are similar in compare with non-pregnant patients, noting that the effect of pregnancy on tumor biology remains until now unclear. Delay in treatment to achieve fetus vitality or improved fetal outcome may be an option for patients with early stage cervical cancer.

Besides surgery, systemic treatment, if compatible with pregnancy, plays an important rolein antenatal oncologic treatment. Neoadjuvant chemotherapy has emerged as an alternative to parallel chemotherapy and radiation therapy, and this may offer patients the opportunity postpones definitive treatment until fetal viability. In this setting based on platinum chemotherapy is the most commonly used systemic therapy in pregnant women. It should be noted that the chemotherapy performed in the second and third trimester may be associated with intrauterine growth retardation, prematurity and low birth weight. In addition, the introduction of chemotherapy within 3 weeks after the intended shipping is not recommended [11]. Our patient, starting from 24 weeks of pregnancy, underwent 3 courses of neoadjuvant cisplatin-containing chemotherapy with an interval of three weeks between courses, with no delay and adverse effects. The last course was completed at 33 weeks of gestation.

There are no studies in the literature on the ideal follow-up option for patients receiving NACT during pregnancy [4]. We performed a follow-up evaluation after completing 3 cycles of NACT, which included pelvic exam, colposcopy and MRI of the pelvic organs. According to these data, almost complete tumor regression was achieved after 3 courses of NACT. A minor residual tumor of the cervix was later confirmed by histological examination.

As far as delivery method is concerned, the literature is mixed. To achieve the ideal approach, several points need to be considered. If possible, delivery should be scheduled by 37 weeks of gestation to avoid prematurity and neonatal complications. Most patients undergo a Caesarean section. The incidence of Cesarean section in pregnant cancer patients is observed in $30 \%$, which is higher compared with a recorded global rate of $21 \%$ $[11,13]$. Vaginal delivery is contraindicated for the most cervical and vulvar cancers because of the potential for implantation of cancer cells in the vaginal tear/episiotomy site [7]. In our patients' case after completed three cycles of neoadjuvant platinum-based chemotherapy at a gestational age of 37 weeks a simultaneous Caesarean section and a radical abdominal hysterectomy were successfully performed.

Cervical cancer diagnosed during pregnancy remains is rare condition [4]. Despite the availability of NCIP recommendations, cancer treatment during pregnancyis still challenging, and decision-making always should be multidisciplinary. If there is a desire to preserve the pregnancy, specialists need to weigh the safety of the mother and child and choose the optimal cancer treat- 
ment possible while minimizing the risks to the fetus and mother $[4,6,7,11]$.

\section{Funding Information}

The author did not receive a specific grant for this research from any funding agency in the public, commercial or not-for-profit sectors.

\section{Authors' Contributions}

O.P.M. was involved in surgical treatment of the patients, analysis of the results and wrote the article.

\section{References}

1. Sonoda K, Ohgami T, Hachisuga M, Fujita Y, Okugawa k, et al. (2021) Difficulty of cervical cancer diagnosis during pregnancy: A case series analysis of the clinicopathological characteristics and prognosis of cervical cancer diagnosed during pregnancy or within 6 months after parturition. Mol Clin Oncol 14: 67.

2. National Comprehensive Cancer Network (NCCN) (2019) NCCN clinical practice guidelines in oncology: Cervical cancer. Version 4.2019-March 29, 2019.

3. Cibula $D$, Potter $R$, Planchamp $P$, Lundqvist $E A$, Fischerova D, et al. (2018) The european society of gynaecological oncology/european society for radiotherapy and oncology/european society of pathology guidelines for the management of patients with cervical cancer. Virchows Arch 28: 641-645.

4. Gomez Rodrigo S, Calderon J, Dionisi JN, Santi A, Mariconde JM, et al. (2021) Cervical cancer in pregnancy at various gestational ages. Int J Gynecol Cancer 31: 784788.
5. Maggen C, Wolters VERA, Cardonick E, Fumagalli M, Halaska MJ, et al. (2020) Pregnancy and cancer: The INCIP project. Curr Oncol Rep 22: 17.

6. Halaska MJ, Uzan C, Han SN, Fruscio R, Steffensen KD, et al. (2019) Characteristics of patients with cervical cancer during pregnancy: A multicenter matched cohort study. An initiative from the International network on cancer, infertility and pregnancy. Int J Gynecol Cancer 29: 676-682.

7. Amant $F$, Berveiller $P$, Boere IA, Cardonick E, Fruscio R, et al. (2019) Gynecologic cancers in pregnancy: Guidelines based on a third international consensus meeting. Ann Oncol 30: 1601-1612.

8. Rabaiotti E, Sigismondi C, Montoli S, Mangili G, Candiani $\mathrm{M}$, et al. (2010) Management of locally advanced cervical cancer in pregnancy: A case report. Tumori 96: 623-626.

9. Swailes AL, Gockley A, Phaeton R, Kesterson JP (2017) The Wertheim hysterectomy: Development, modifications, and impact in the present day. Gynecol Oncol 145: 3-8.

10. Floyd JL, Campbell S, Rauh-Hain JA, Woodard T (2021) Fertility preservation in women with early-stage gynecologic cancer: Optimizing oncologic and reproductive outcomes. Int J Gynecol Cancer 31: 345-351.

11. Wolters V, Heimovaara J, Maggen C, Cardonick E, Boere I, et al. (2021) Management of pregnancy in women with cancer. International Journal of Gynecologic Cancer 31: 314-322.

12. Peculis LD, lus $Y$, Campion $M$, Friedlander $M$, Hacker $N$ (2015) Stage IB2 adenosquamous cervical cancer diagnosed at 19-weeks' gestation. Aust N Z J Obstet Gynaecol 55: 94-97.

13. Lees C, Marlow N, Arabin B, Bilardo CM, Brezinka C, et al. (2013) Perinatal morbidity and mortality in early-onset fetal growth restriction: Cohort outcomes of the trial of randomized umbilical and fetal flow in Europe (TRUFFLE). Ultrasound Obstet Gynecol 42: 400-408. 